\title{
A TWO-CHANNEL EEG RADIO TELEMETERING SYSTEM
}

\author{
A. Kamp and W. Storm van LeEuwen \\ Institute of Medical Physics TNO, Utrecht (The Netherlands)
}

(Received for publication: February 2, 1961)

In EEG the electrodes are usually connected by means of wires to the terminal box of the EEG apparatus. For this reason movements of the subject are restricted and, moreover, movements of the connecting wires may cause artefacts. In some investigations in man as well as in animals it may be necessary, however, to record the
For recording the electrical activity of the brain in a normal, free, and unrestrained cat, Gold and Perkins (1959) developed a miniature telemetering system.

Vreeland et al. (1958) constructed a miniature transistor telemeter similar to the vacuum tube unit described by Glasscock and Holter (1952). Shipton recently (1960)

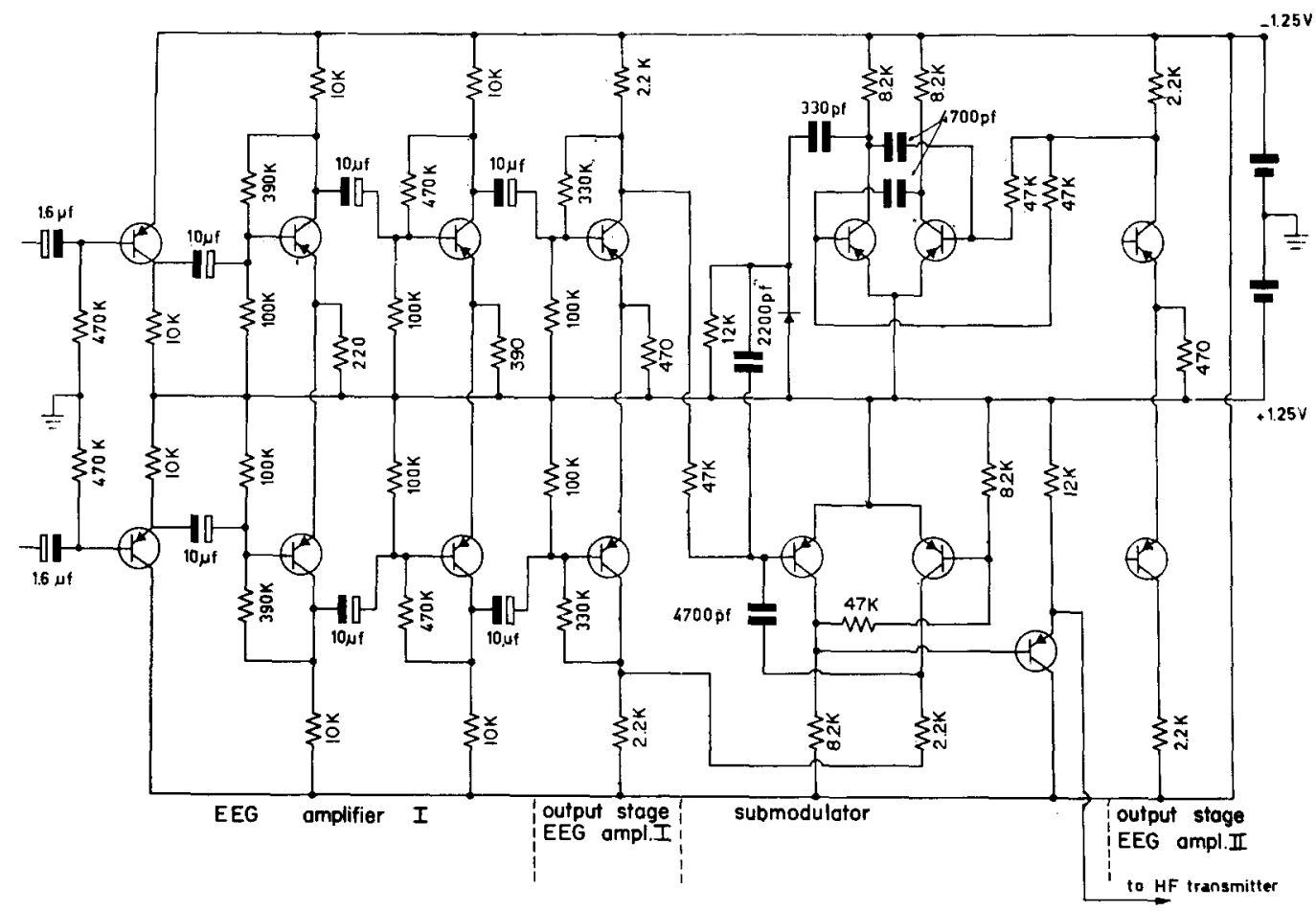

Fig. 1

Diagram of the EEG amplifiers and sub-modulator.

EEG while the subject is moving. In these cases wireless transmission of the EEG can be used. This method implies that the subject has to carry the amplifying and transmitting apparatus, and requires that the dimensions and the weight of this apparatus should be as small as possible.

Applications of wireless transmission of the EEG have already been published by various investigators. Glasscock and Holter (1952) and Götze et al. (1958) described portable apparatus for EEG telemetering employing vacuum tube circuits. described a system for telemetering electrophysiological data. These devices are not well suited for multi-channel EEG transmitting because of the weight of the amplifiers, of inadequate amplification, or of inappropriate input impedances and time constants. In comparison with vacuum tubes, transistors allow a considerable decrease in the dimensions and weight of the apparatus and thus offer the possibility of increasing the number of channels to be recorded.

For this reason a two-channel transistor telemetering 
system has been developed. The apparatus consists of two identical amplifiers, a sub-modulator and an HF transmitter. Each of the four stage EEG amplifiers has a gain of 3000 . Emitter-follower input stages provide for an the HF oscillator. The oscillator has a center frequency of $52 \mathrm{Mc}$. The tank circuit in the collector lead of the power amplifier is tuned to twice the frequency of the oscillator. Thus the transmitter signal has a center fre-

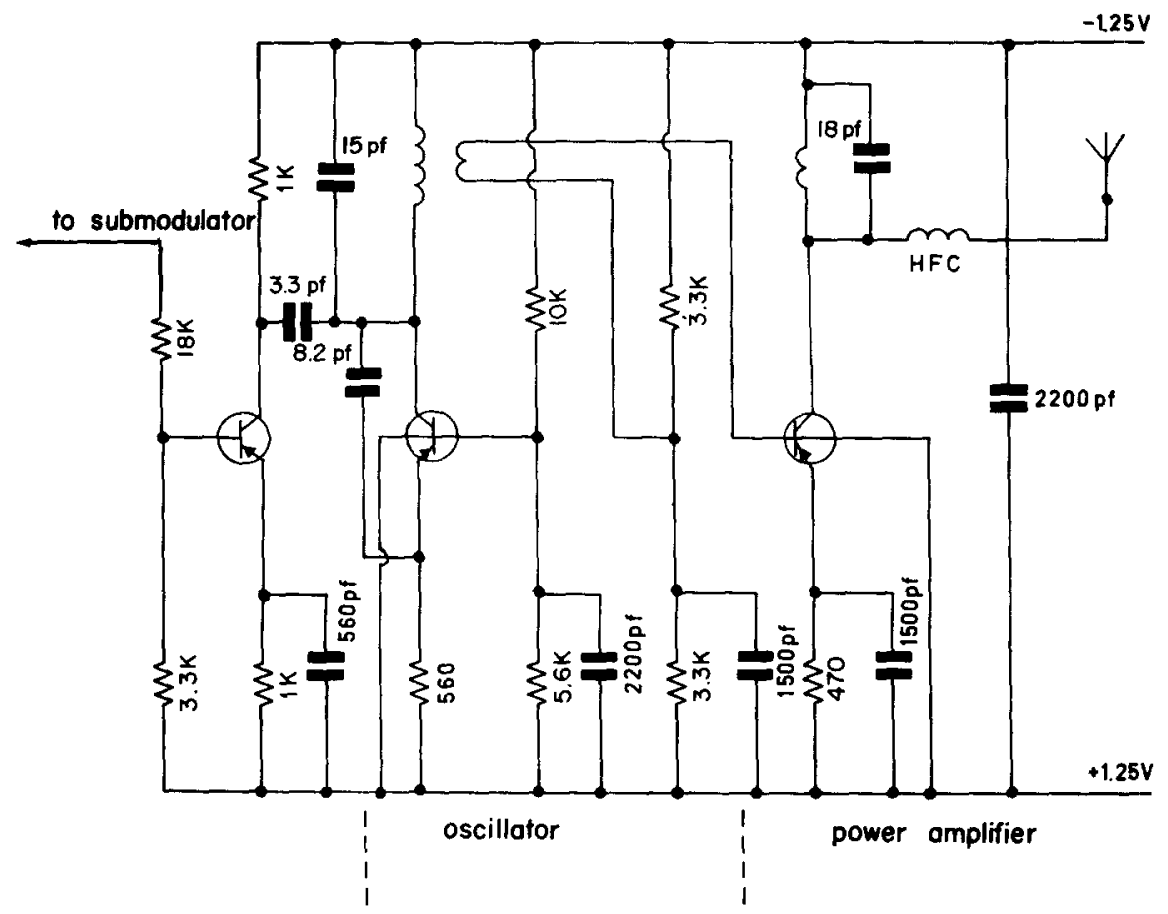

Fig. 2

Diagram of the HF transmitter.

input impedance of $300 \mathrm{k} \Omega$. The transistors of the input stages are selected for minimum noise, and to obtain the required gain, transistors having $\beta>100$ are used.Alloyed junction high frequency transistors have been used for the amplifier stages since with these transistors a current amplification of about 100 can be obtained with a reasonably low zero collector current $\mathrm{Ic}_{0}$. The low $\mathrm{Ic}_{0}$ permits adjustment to a low collector current, ensuring minimal current consumption from the batteries. Fig. 1 shows a complete diagram of the amplifiers and the connections of the two amplifier output stages with the sub-modulators. These submodulators perform combined pulse-frequency and pulse-duration modulation. The outputs of the two amplifiers are connected to a multi-vibrator and a monostable trigger. The pulse-duration of the monostable trigger varies linearly with the output voltage of amplifier I. The output voltage of amplifier II effects frequency modulation of the multi-vibrator square wave. As the monostable trigger is started by the multi-vibrator, the output pulses of the monostable trigger vary in duration as well as in repetition rate in accordance with the EEG amplifier output voltages. The output pulses provide for frequency modulation of the HF oscillator in the transmitter part.

Fig. 2 shows the diagram of the HF transmitter. Frequency modulation is effected by the variation of the collector capacitance which shunts the tuning capacitor of quency of $104 \mathrm{Mc}$, and the deviation is $75 \mathrm{Kc}$. Power is supplied by two $1.25 \mathrm{~V}$ miniature storage batteries with a capacity of $150 \mathrm{mAh}$. The total current consumption from the batteries is $7 \mathrm{~mA}$ and thus the apparatus can te used for about $20 \mathrm{~h}$ without charging the batteries.

As the radiated power is very small, it is necessary to use three separate receivers. The aerials of these receivers have different directional sensitivities. With these provi-

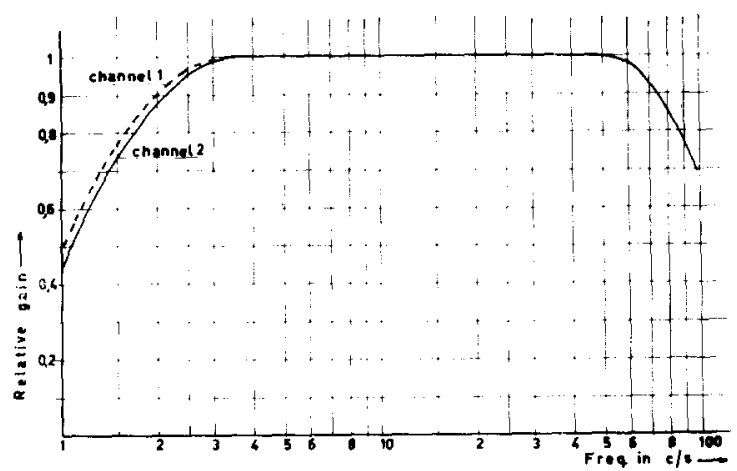

Fig. 3

Frequency response of the complete system. 
sions the telemetered signals can be received in an area with a radius of about $30 \mathrm{~m}(100 \mathrm{ft})$. The cross-talk between the two channels is less than 3 per cent.

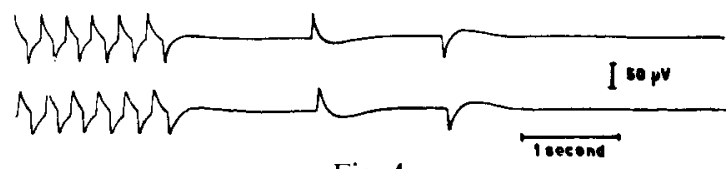

Fig. 4

Calibration signal of $50 \mu \mathrm{V}$.
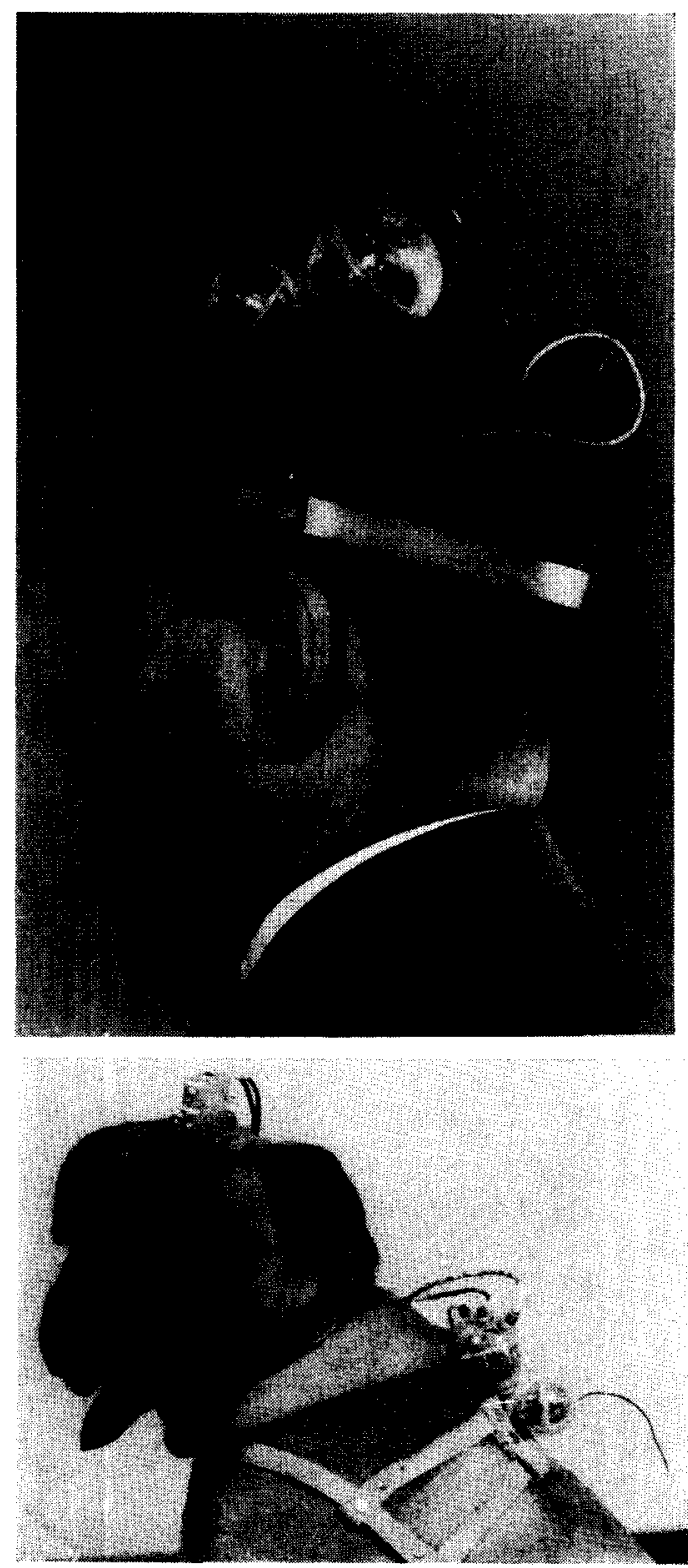

Fig. 5

Radio-telemeter for transmitting human EEG, (above) and EEG in dogs (below).
The receiver may be any commercial FM receiver. Feedback from the receiver ratio-detector to the oscillator provides for automatic tuning of the receiver.

Movement of the transmitter with respect to the body of the subject (human or animal) may cause frequency variations of the transmitter oscillator. Such frequency variations are compensated by automatic tuning of the receiver.

The output pulses of the ratio detector are similar to those which provide for frequency modulation in the transmitter part.

The EEG of channel $\mathrm{I}$ is obtained by measuring the peak voltage of a capacitor which is periodically charged during a time, equal to the duration of the ratio detector output pulses. The leading edge of these pulses also starts a monostable trigger. Integration of the monostable trigger output voltage by means of a lowpass filter gives the EEG of channel II.

Fig. 3 shows the frequency response of the complete system measured from the amplifier input to the recording

A

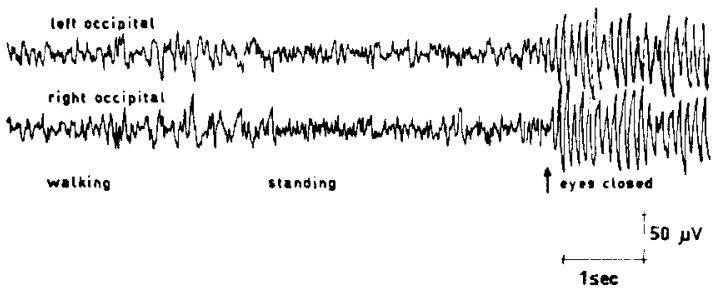

1

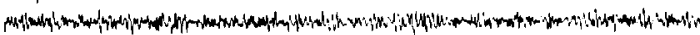

2

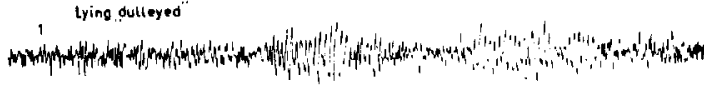

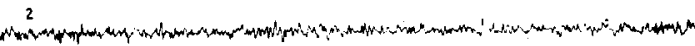

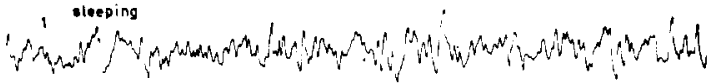

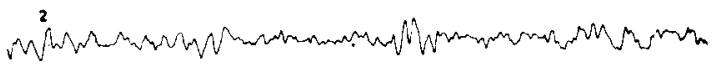
isec

Fig. 6

A. EEG of a normal subject while walking and standing with eyes open and while standing with eyes closed.

B. EEG of a dog when walking, when lying "dull eyed", and when asleep.

1. Derivation from chronic implanted depth-electrode (probably hypothalamic).

2. Derivation from cranial electrodes (occipito-parietal.) 
apparatus. With a $10 \mathrm{k} \Omega$ resistance across the amplifier inputs, the equivalent noise level of the entire system is approximately $2 \mu \mathrm{V}$ (Fig. 4).

The total weight of amplifiers, transmitter, and batteries is $100 \mathrm{~g}$. In Fig. 5A the apparatus as used in investigations on humans is shown.

Fig. 5B demonstrates an adaptation of the apparatus for correlation between electrical activity of the brain and behaviour in dogs. Results obtained in the human subject are shown in Fig. 6A. Note absence of movement artefacts when subject is walking. In Fig. $6 \mathrm{~B}$ are shown curves obtained in a dog from a depth derivation 1 (probably hypothalamic) and from a surface derivation 2 (parietooccipital cranial electrodes). Note absence of movement artefacts when animal is walking, increase in depth lead of $18-20 \mathrm{c} / \mathrm{sec}$ activity when animal is lying down, awake but looking "dull eyed," and increase of slow activity when animal is asleep.

\section{REFERENCES}

Glasscock, W. R. and Holter, N. J. Radio electroencephalograph for medical research. Electronics, 1952, 25: $126-129$.

Götze, W., Kofes, A., Kubicki, S. T. und Wolter, M. EEG- und EMG-Untersuchungen an Gesunden und Hirnkranken unter Arbeitsbelastung nach Radioübertrachung. Elektro Med., 1958, 3: 297-303.

Gold, D. C. and Perkins, W. J. A miniature electroencephalograph telemeter system. Electronic Eng., 1959, 31: 337-339.

Vreeland, W. R., Williams, L. A., Yeager, C. L. and Henderson, J. Unit telemeters scalpvoltages. Electronics, 1958, 3I: 86.

Shipton, H. W. A simple telemetering system for electrophysiological data. Electroenceph. clin. Neurophysiol., 1960, 12: 922-924.

Reference: Kamp, A. and Storm VAn Leeuwen, W. A two-channel EEG radio telemetering system. Electroenceph. clin. Neurophysiol., 1961, 13: 803-806. 